\title{
AToMRS: A Tool to Monitor Recommender Systems
}

\author{
André Costa ${ }^{1}$, Tiago Cunha ${ }^{1}$ and Carlos Soares ${ }^{1}$ \\ Faculdade de Engenharia da Universidade do Porto, Porto, Portugal \\ \{ee07285, tiagodscunha, csoares\}@fe.up.pt
}

Keywords: Recommender Systems, Collaborative Filtering, Evaluation.

\begin{abstract}
Recommender systems arose in response to the excess of available online information. These systems assign, to a given individual, suggestions of items that may be relevant. These system's monitoring and evaluation are fundamental to the proper functioning of many business related services. It is the goal of this paper to create a tool capable of collecting, aggregating and supervising the results obtained from the recommendation systems' evaluation. To achieve this goal, a multi-granularity approach is developed and implemented in order to organize the different levels of the problem. This tool also aims to tackle the lack of mechanisms to enable visually assessment of the performance of a recommender systems' algorithm. A functional prototype of the application is presented, with the purpose of validating the solution's concept.
\end{abstract}

\section{INTRODUCTION}

Currently there is a high demand for internet services for both personal and professional reasons. This growth in demand has also fueled the amount of information available to meet the user needs. However, the scale of available data has prevented the easy access to the relevant information. To tackle this problem, Recommender Systems (RSs) have been developed to personalize the user experience in the demand for relevant information. This allowed the reduction of the user's query effort, while enabling the enterprises easier promotion of their products (Wei et al., 2007).

The success of RS depends on the user experience. This experience is, among other factors, dependent on the RS performance (Herlocker et al., 2004). Therefore, researchers have developed several evaluation metrics to estimate the RS performance in order to approximate the user experience. Unfortunately, RSs are not infallible and it is of importance to continuously evaluate their performance in order to enforce the quality required (Tintarev and Masthoff, 2011).

The evaluation process of RS is not a simple task, since there are several problems that must be dealt with. On one hand, it is essential to choose appropriate data sampling strategies, algorithms, metrics and evaluation procedures to ensure a valid evaluation procedure. On the other, it is also important to allow a continuous evaluation of the RS performance to assess if the performance deteriorates across time.

In order to do so, a multi-granularity approach is proposed to extend the current global evaluation procedure towards a more fine grained analysis. This means that instead of solely providing the average performance results that a recommendation algorithm obtains on the entire dataset, we split the data into different layers and report the performance values of all metrics on said layers. The layers used are: (1) the dataset, (2) the data batch and (3) the user-item relationship. The data batch is derived from the data sampling strategy used to evaluate the RS. Although in this work the focus lies on the analysis of batches as each fold of the cross-validation procedure, the approach is designed to also handle online evaluation procedures. The idea is to use the incremental data to train and evaluate the models through a new batch.

Despite the existence of several evaluation platforms for RS, none is found that allows such an "atomic" analysis of the recommendation problem. And very few provide an intuitive graphical visualization tool to further help the RS practitioner to monitor its performance. Therefore, this paper also presents the prototype developed to establish proof of concept.

This paper is organized as follows: Section 2 presents the related work on RS and platforms to evaluate and monitor their performance. Section 3 presents the multi-granularity approach and the prototype. On Section 4 the entire proposal is discussed and Section 5 presents the conclusions of the study and highlights tasks for future work. 


\section{RELATED WORK}

\subsection{Recommender Systems}

RS emerged with the goal to help users navigate through high volumes of information, by preparing personalized recommendations, content and services (Adomavicius and Tuzhilin, 2005). RS gather data regarding the user preferences towards the items (Bobadilla et al., 2013) and use Machine Learning methods in order to provide the recommendations (Bagchi, 2015). In this context, the item can be anything that the system recommends (for instance, movies, music, ...) (Tintarev and Masthoff, 2011).

In the recommendation process, it is imperative to take into account (1) the type of data available, (2) the suitable recommendation techniques for the collected data, (3) the recommendation algorithms, (4) the recommendation target and (5) the scope of evaluation desired (Bobadilla et al., 2013). In this work the focus lies on the Collaborative Filtering (CF) recommendation technique. Thorough surveys are available for the remaining techniques (Adomavicius and Tuzhilin, 2005; Bobadilla et al., 2013).

\subsubsection{Data}

The data used in RS are mostly related to the user, the item and the relationships between them (Tintarev and Masthoff, 2011). Although there is a large variety of data useful for RS, in CF the data is simply a value of preference that the user assigned the item. This preference can be explicit (a numerical rating) or implicit (unary or binary variable that ascertains the interest of the user on the item) (Tintarev and Masthoff, 2011).

The data structure used in CF is known as the rating matrix $R$. It is described as $R=U \times I$, representing a set of users $\mathrm{U}$, where $u \in\{1 \ldots N\}$ and a set of items $\mathrm{I}$, where $i \in\{1 \ldots M\}$. Each element of this matrix is the numerical feedback provided by a user $u$ relative to an item $i$, represented by $r_{u i}$.

\subsubsection{Strategy}

CF methods are organized into memory-based and model-based. While memory-based methods act only on the rating matrix using heuristics to obtain the recommendations, model-based methods induce a model with the data available to provide the recommendations (Su and Khoshgoftaar, 2009). Usually, memorybased methods are based on nearest neighbors and model-based methods on matrix factorization.

Nearest neighbor strategies have three main steps: (1) calculate similarity among users/items, (2) finds the $k$ most similar neighbors and (3) predict the items to be recommended accordingly to the preference values of neighbors (Su and Khoshgoftaar, 2009). Several similarity metrics can be used in the process. Some common metrics are Pearson correlation and Cosine similarity (Sarwar et al., 2001).

Matrix factorization methods approximate the rating matrix values by the multiplication of at least two matrices with latent features that capture the underlying data patterns (Koren, 2010). The computation is iterative and optimizes an error metric. There are several matrix factorization methods (Singular Value Decomposition, Alternating Least Squares, Stochastic Gradient Descent) (Hu et al., 2008).

\subsubsection{Algorithms}

There are several recommendation frameworks available. Due to its extensive amount and diversity of CF methods, we chose the MyMediaLite (MML) framework (Gantner et al., 2011). The algorithms are organized into two major CF tasks: Rating Prediction (RP) and Item Recommendation (IR). Although the data used is the same, the recommendation process is different. The discussion of the algorithms falls outside the scope of this paper.

Nearest neighbor methods are available to both RP and IR and are called ItemKNN and UserKNN. The similarity metrics available are Pearson's correlation, Cosine similarity, Jaccard coefficient, Conditional probability, Bi-conditional probability and $\mathrm{Co}-$ occurence.

Matrix Factorization methods are divided into RP and IR. MML presents a total of 7 algorithms for RP and other 7 for IR. Despite sharing the same nature, the processes used are different. The discussion of these methods is not approached in this document, but they are presented in Table 1.

The baseline algorithms included in MML for RP are the GlobalAverage, UserAverage, the Item Average and the UserItemBaseline. For IR, the only baseline is MostPopular.

\subsubsection{Evaluation}

As was stated previously, the evaluation process in RS is crucial and it must happen in several stages of the system's life cycle. During its development, the RS must be validated to ensure that the best recommendation algorithm was chosen. This is achieved through offline evaluation procedures, where several algorithms are compared on the same dataset (Tintarev and Masthoff, 2011). Afterwards, it is also critical to evaluate the RS performance when it is online. Here, 
Table 1: Matrix Factorization methods in MyMediaLite

\begin{tabular}{|c|c|}
\hline Algorithm & Type \\
\hline Matrix Factorization & RP \\
\hline BiasedMatrixFactorization & RP \\
\hline LatentFeatureLogLinearModel & RP \\
\hline SVDPlusPlus & RP \\
\hline SigmoidItemAsymmetricFactor & RP \\
\hline SigmoidUserAsymmetricFactor & RP \\
\hline SigmoidCombinedAsymmetricFactor & RP \\
\hline BPRMF & IR \\
\hline BPRSLIM & IR \\
\hline LeastSquareSLIM & IR \\
\hline MultiCoreBPRMF & IR \\
\hline SoftMarginRankingMF & IR \\
\hline WeightedBPRMF & IR \\
\hline WRMF & IR \\
\hline
\end{tabular}

real users feedback is used to compute online evaluation metrics. Both evaluation strategies should ideally be performed, since it does not necessarily mean that a good offline performance will yield a good online performance (Tintarev and Masthoff, 2011). However, this is sometimes unattainable due to the effort required to collect online user feedback. In this work the focus lies on offline evaluation, but prepares the work for online evaluation.

In offline evaluation, the dataset must be partitioned into training and test datasets. This is achieved by splitting the rating matrix into different sets of users and use their observations to either train or test the RS model (Hahsler, 2011). There are several data partitioning methods available: split, hold-out, leaveone-out and k-fold cross-validation.

Afterwards, the algorithm is trained on the training data and its predictions are compared to the hidden values from the testing dataset. This comparison is performed by using several evaluation metrics. The evaluation metrics available are: (1) predictive precision, (2) classification precision and (3) ranking precision (Herlocker et al., 2004).

Predictive precision measures how close is the prediction to the actual user rating. It is based on error metrics, such as Mean Average Error (MAE) and Root Mean Squared Error (RMSE). Classification precision measures the frequency of correct decisions made by the RS regarding the item utility. The metrics are based on standard classification precision metrics such as precision, recall and accuracy. Ranking precision metrics are used to measure an algorithm's ability to produce an ordered list of items that match the order the user would have chosen (Herlocker et al., 2004). The standard metrics for this task are Means Average Precision (MAP), Normalized Discount $\mathrm{Cu}-$ mulative Gain (NDCG) and Mean Reciprocal Rank (MRR). Classification precision metrics can also be adapted to ranking problems by selecting the top $\mathrm{N}$ elements of both the training and testing datasets, instead of the entire set of items available (Herlocker et al., 2004).

\subsection{Recommendation Platforms}

From the large amount of recommendation platform, this work highlights the most popular: Apache Mahout (Owen et al., 2011), LensKit (Ekstrand et al., 2011) and MyMediaLite (MML) (Gantner et al., 2011). Despite containing approximately the same recommendation algorithms, there are differences regarding the data partitioning and evaluation procedures.

Apache Mahout contains a large amount of recommendation algorithms and provides distributed processing for a few of them. However, the evaluation procedure is poor: there is no cross-validation procedure and the evaluation metrics are limited to the classification precision category. Lenskit provides only basic algorithms and the metrics are based on predictive precision. However, it allows a more suitable evaluation procedure by providing cross-validation. Lastly, MML arises as the more complete platform: it contains the largest amount and variety of recommendation algorithms, several data partitioning techniques (including cross-validation) and provides suitable metrics for each $\mathrm{CF}$ task: predictive precision metrics for RP and ranking precision metrics for IR.

A comparison of these platforms regarding these three platforms is available (Said and Bellogín, 2014). The study concludes that despite providing the most computational resource consuming algorithms, MML provides a superior performance. Further information regarding the comparison of these platforms is presented in Table 2.

Table 2: Comparison of recommendation frameworks.

\begin{tabular}{|l|c|c|c|}
\cline { 2 - 4 } \multicolumn{1}{c|}{} & Mahout & MML & LensKit \\
\hline Release date & $04 / 2007$ & $10 / 2010$ & $03 / 2011$ \\
\hline Last update & $05 / 2015$ & $12 / 2015$ & $11 / 2015$ \\
\hline Last version & 0.10 .1 & 3.11 & 2.2 .1 \\
\hline Language & Java & C\# & Java \\
\hline Platform & JVM & .NET & JVM \\
\hline Algorithms & Classical & SotA & Classical \\
\hline Distributed & Partial & No & No \\
\hline
\end{tabular}




\subsection{Monitoring Platforms}

Despite the existence of several evaluation platforms with integrated evaluation procedures, there are few monitoring platforms for RS.

A widely used tool to store, distribute and analyze Data Mining experimental data is OpenML (Vanschoren et al., 2014). The repository is important mainly for offline evaluation procedures, since several researchers can provide their experimental data for the same problem and compare the results with others. There are also several visualization mechanisms to perform an analysis of the gathered experimental data and it allows an open and collaborative discussion of the results obtained. However, this work is not specifically focused on RS and it is not prepared for a proper online monitoring of the recommendation process.

One tool available in the literature regarding RS monitoring for music recommendation (Félix et al., 2014). The RS provides recommendations for the users, who are responsible to either blacklist them or to add the musics to the library. Based on this feedback, the system performs online evaluation procedures. The system monitors the positive and negative feedback provided and analyses it through several facets: date, time of day, user gender and geographical distribution. These results are provided via visualization mechanisms to better understand the previous facets. The mechanisms allow the RS practitioner to adjust the RS in order to maximize the effectiveness of the recommendation process. The main difference to the work proposed in this paper is the level of analysis that the system allows. The multi-granularity approach proposed will allow a deeper analysis of the recommendation problem.

A platform for Website automation and monitoring is also available (Domingues et al., 2008). The platform aims to generically maintain Web pages via Web adapters. The case study presented uses one of these adapters as a RS and its performance is monitored continuously. The developed monitoring tool, EdMate, takes advantage of online evaluation metrics and displays the information via numerical and graphical mechanisms. The tool reports statistics regarding the website usage, the recommendation adhesion from the users, the recommendation efficacy and the time spent by the users on specific pages. Despite providing an extensive overview of the recommendation process, this tool focus on analyzing several indicators. The proposed approach in this paper, focuses instead on providing a deeper analysis to the standard evaluation procedure.

\section{AToMRS}

\subsection{Multi-granularity approach}

The approach used in this work to enable a deeper analysis of the RS evaluation procedure has its foundation in Machine Learning data splitting strategies used to train and test models. Figure 1 presents the several levels that can be analyzed.

The process starts in the dataset level, with all the available instances. Then, data is split to create the training and testing datasets. The data splitting strategy creates data batches, which can be used to analyze different sub-problems of the original recommendation problem. In the approach designed here, the batches are folds in the cross-validation. Lastly, the user-item $<u, i>$ interaction level presents a single instance of the original dataset. These are fundamentally different for the specific CF strategy: in $\mathrm{RP}$, the association is defined by a triplet user, item and rating value (i.e., $\langle u, i, r\rangle$ ), while in IR a list $l=\left[i_{1}, i_{2}, \ldots, i_{N}\right]$ of ordered items is assigned to each user (i.e., $<u, l>$ ).

All these different facets of the original dataset provide the different levels of analysis of the evaluation procedure: the entire dataset, the data batch and the user-item interaction.

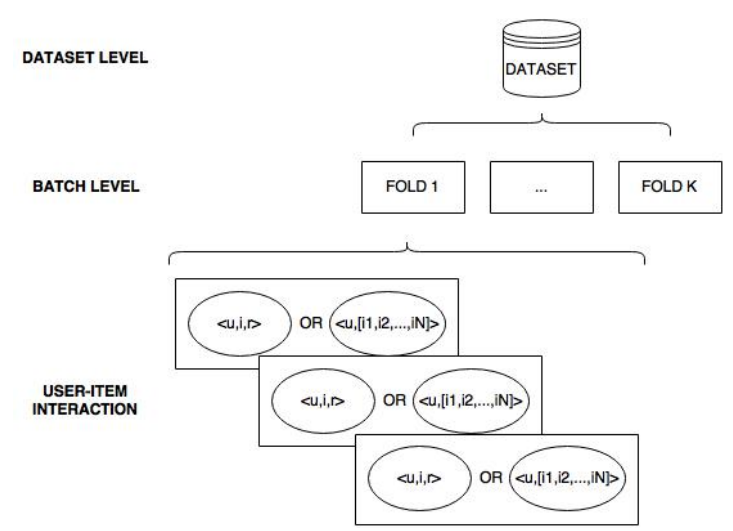

Figure 1: Different levels of the multi-granularity approach.

Despite modeling the data batch as a fold of the offline cross-validation procedure, it is straightforward to adapt the concept to online evaluation: the data batches can be defined by incremental data. Each new incremental dataset is considered a new batch to the problem. Here, the focus is to understand how new data changes the RS performance in several levels of analysis, instead of analyzing the different sub-dataset used to train and validate the model. However, both tasks are of the utmost importance and the approach developed enables both seamlessly. 


\subsection{Data model}

Since the multi-granularity approach developed is based simply on data splitting techniques, the implementation of said approach is simply focused on the design of a suitable database structure. The developed database structure is presented in Figure 2. Please note that the schema follows the NoSQL methodology provided by Apache Cassandra.

For the CF problem, 3 tables were required due to the differences in RP and IR tasks. One main table Experiments describes the general information regarding the $\mathrm{CF}$ experiments and two tables refer to the detailed data for the RP and IR tasks (UItem_Rating for RP and UItem_Recom for IR).

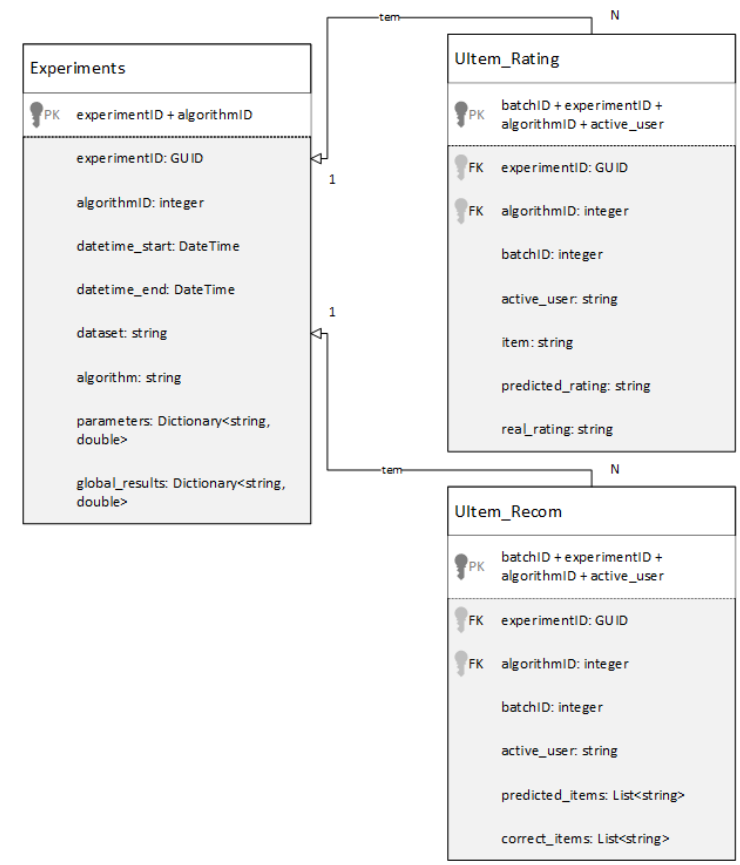

Figure 2: Data model for multi-granularity approach.

The Experiments table stores the global performance results, the start and end dates, the dataset, the algorithm and its parameters. In this table, the experiment and algorithm identifier code are used as the primary key. The remaining tables, which are related to the Experiments table, define the batch and the active user identifier codes.

However, due to the data structures used in each CF task, there are differences in the remaining fields. While UItem_Rating contains the item identifier code and both the real and predicted ratings, in UItem_Recom the data stored are simply two lists: the original and predicted item preference lists.

Data is stored in such way to enable to access each level of the multi-granularity approach. This means that if the target are the user-item interactions, then these can be directly loaded from the database. If, for instance, the goal is to analyze a batch, then data is queried by a specific batch and joined to produce the final results. Lastly, to analyze all instances of a dataset, the query retrieves all instances of that dataset.

This allows to apply the evaluation metrics dynamically to each set of instances upon request on the prototype. The evaluation metrics implementation are common for both the experimental work and for the prototype visualization mechanisms.

\subsection{Prototype}

The prototype was developed as proof of concept for the multi-granularity approach proposed. It has two main modules: the recommendation framework and the application. The architecture diagram is presented in Figure 3.

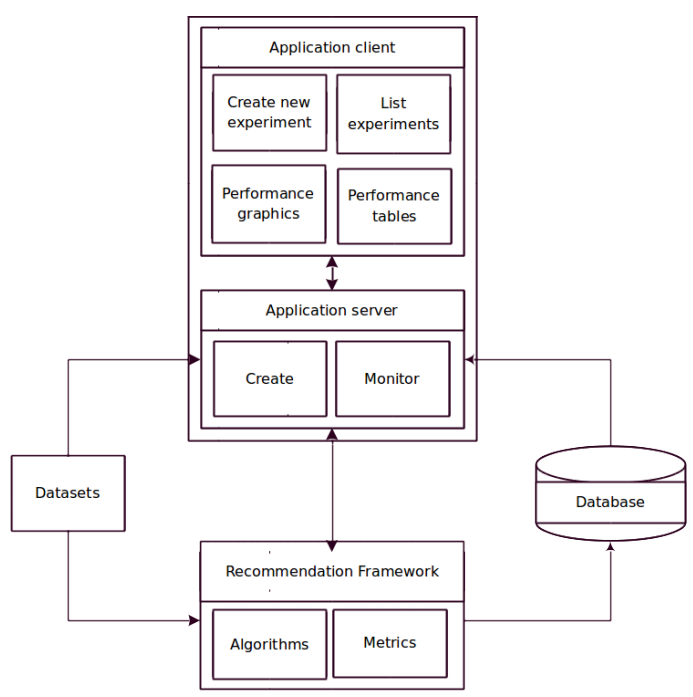

Figure 3: System architecture.

The recommendation framework provides an interface to MML and allows to create the CF experiments. This interface was created by manipulation of the original MML source code. To create an experiment there are a few items to be addressed: the dataset, the algorithms and the respective parameters. At this point, only the cross-validation is enabled, but future work will include other validation strategies.

The evaluation procedure is performed using internal MML data structures and methods. The results from the experimental work are continuously being stored in the database. These can be queried in the 
future by the application to enable the analysis of results.

The application provides a dashboard and several visualization mechanisms that provide access to the several levels of the multi-granularity approach. The dashboard presents management options for experiments (list, create and show). When a specific experiment is selected, then all the dataset level metrics are provided. By selecting a different metric, the results are updated accordingly. Figure 4 presents this interface.

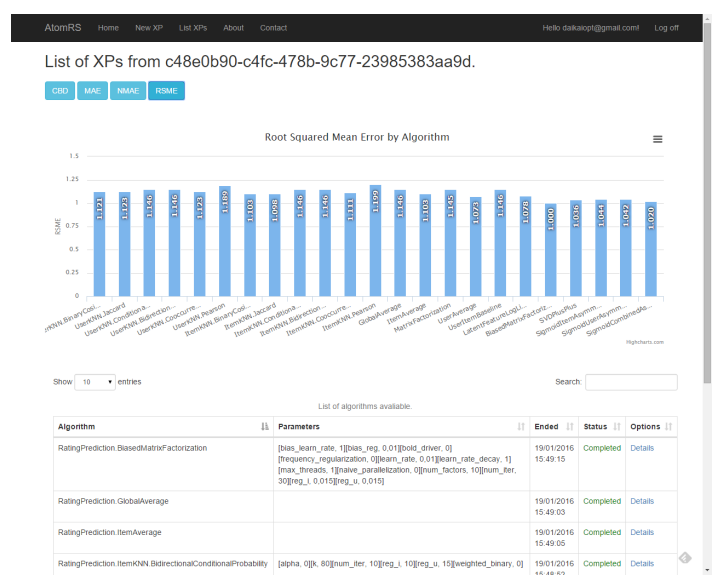

Figure 4: Prototype dashboard.

The dataset level performance is displayed via a bar graphic. The values are shown for a specific metric and it allows to compare the average performance of all algorithms used in the experiment. Figure 5 presents the respective visualization mechanism. Here, the horizontal axis present the several algorithms evaluated on a specific dataset and the vertical axis the corresponding performance metric value.

It is also possible to analyze the results on a batch level. One must select which algorithm is the target for evaluation, and a list of all the batches performance for the specific algorithm are provided. Here, each bar in the graph represents a batch and the vertical axis presents the respective performance metric value. This allows to understand how the performance varies depending on the batch. This representation is also useful for the online evaluation paradigm. Figure 6 presents this visualization mechanism.

On the atomic level, i.e. the user-item interaction, the visualization mechanism is different: instead of using a bar graphic, the performance values are presented via a point dispersion graph with 3 axis $(x, y, z)$ representing user, item and performance value. In this representation, if the values are superior to a prespecified threshold, a different color is assigned to better distinguish the good from the bad results. Figure 7 displays this visualization mechanism.

\section{DISCUSSION}

The presented approach provides a deeper analysis of the CF evaluation procedure, when compared to other monitoring platforms. Furthermore, the work focuses on a new perspective of data analysis, forgotten so far by related works in RS. This section discusses several aspects of both the multi-granularity approach and the prototype.

In terms of the proposed approach to analyze the recommendation problem on several levels, there are two main points to discuss: the data batch structure and the database usage.

While the data batch structure served its purpose and enabled all the work developed, it has low scientific value. Its merits lie on a more practical perspective. However, it is important to notice that this structure can be re-used (and even improved) for other Data Mining tasks that require low level analysis.

Secondly, the proposed method requires high computational storage resources, especially if the dataset scales dramatically. Also, in terms of computational time, the approach introduces a significant overhead. This overhead is well worth if the dataset used is small, because of the deeper analysis power enabled. But it may become overwhelming and impracticable if the data scale increases beyond reasonable values.

Regarding the prototype, the discussion points highlighted are the visualization mechanisms and the adaptation towards online evaluation.

The visualization mechanisms use mostly the bar graphics to represent the performance values. The authors believe that visualization mechanisms are suitable for the task at hand. On one hand, they present an intuitive way to evaluate the performance results and, on the other, it fits directly to any level described by the data model. Therefore, by reusing the same visualization mechanism, it promotes stability and does not introduce a learning curve for the user. However, this is a debatable position: it is possible that many other visualizations with added value can also be included. But the trade-off between usability and ease of analysis needs to be taken into account.

The online evaluation adaptation, although taken into consideration when designing the solution, is not implemented yet. This requires a functional RS that can receive feedback from real users. However, it is the authors belief that on a practical perspective, the problem can be modeled in a similar fashion. If so, then the same solution developed can be directly applied. 


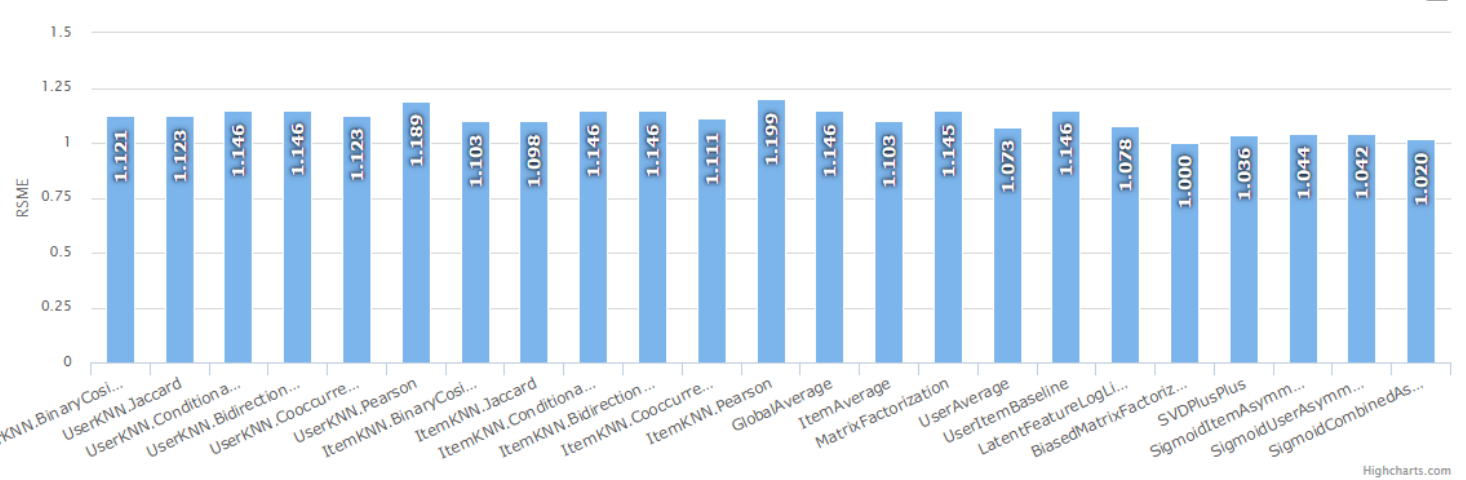

Figure 5: Global performance values on dataset level.

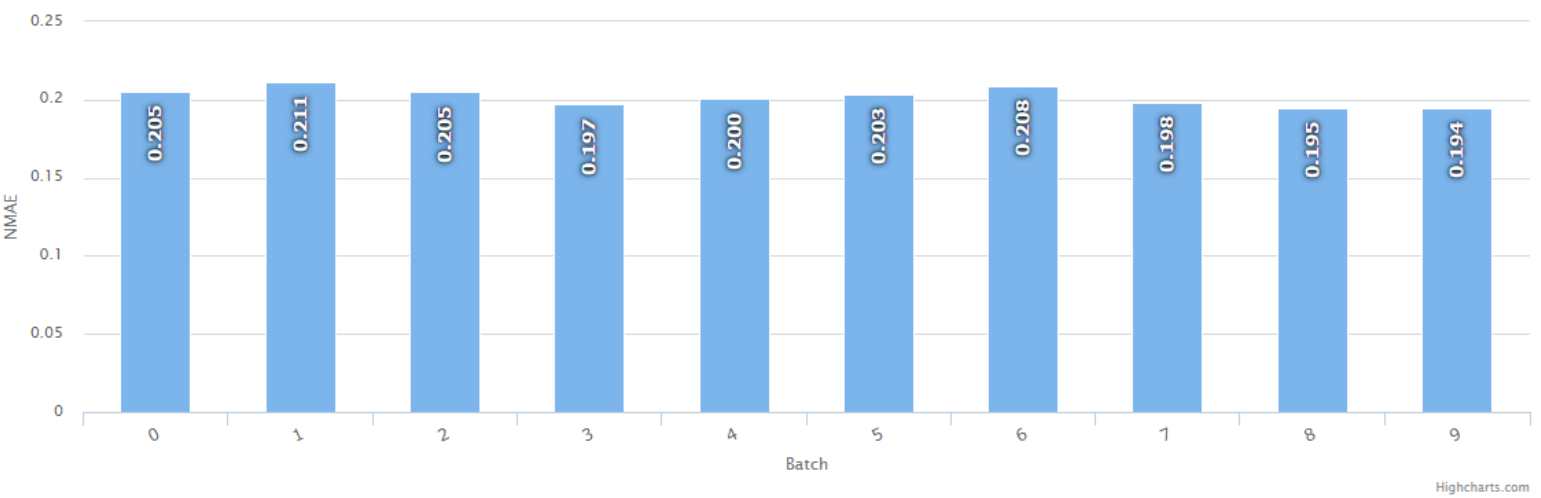

Figure 6: Batch level performance values across batches.

Singular errors for the \#6 batch

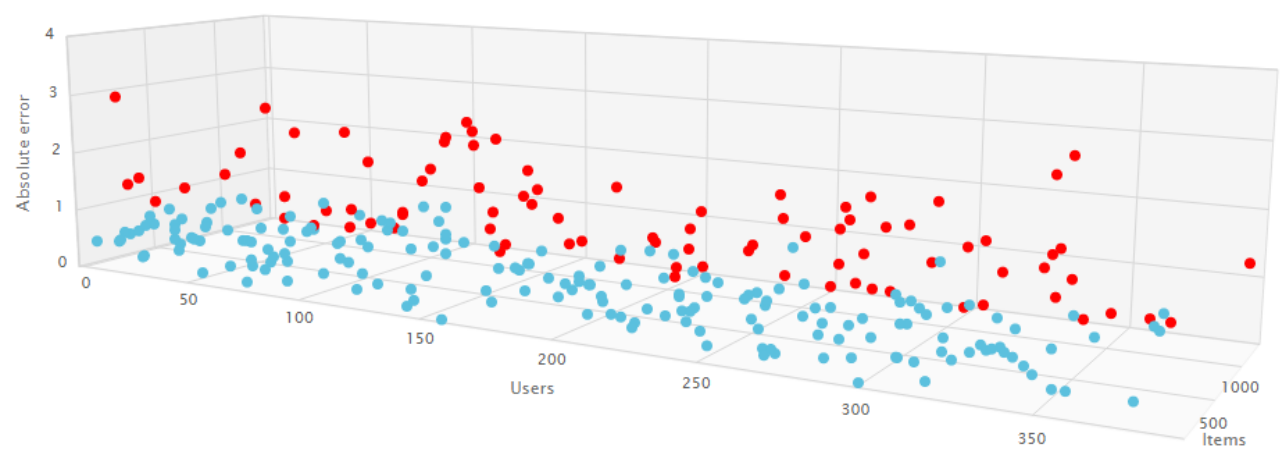

Figure 7: User-item level performance values for a specific batch. 


\section{CONCLUSIONS}

This work proposed a multi-granularity approach to allow a deeper analysis of a RS performance. This approach allows to analyze the recommendation problem in 3 levels: the dataset, the batch and the useritem interaction. This was achieved via a database model that enables the storage and access of this hierarchical data. In this work's case study, the approach was applied to offline evaluation, although it was designed to also handle the online evaluation procedure.

A prototype was also developed to demonstrate the applicability of the approach designed. The prototype is responsible for experiment management operations of the recommendation framework MyMediaLite. It also displays the performance results of the multi-granularity approach using several mechanisms.

Tasks for future work include an optimization of the experiment management options (include setting algorithm's parameters, perform grid search, schedule experiments), extend the work on visualization mechanisms (study other appropriate visualization representations for the developed multi-granularity approach) and create a case study for online evaluation to validate the assumptions stated in this work.

\section{ACKNOWLEDGEMENTS}

This work is financed by the ERDF Fund through the Operational Programme for Competitiveness and Internationalization - COMPETE 2020 of Portugal 2020 through the National Innovation Agency (ANI) as part of the project 3506 and also through project POCI-01-0145-FEDER-006961 via National Funds through the FCT - Fundação para a Ciência e a Tecnologia as part of project UID/EEA/50014/2013. The research was also funded from the ECSEL Joint Undertaking, the framework programme for research and innovation horizon 2020 (2014-2020) under grant agreement 662189-MANTIS-2014-1.

\section{REFERENCES}

Adomavicius, G. and Tuzhilin, A. (2005). Toward the next generation of recommender systems: A survey of the state-of-the-art and possible extensions. IEEE Transactions on Knowledge and Data Engineering, 17(6):734-749.

Bagchi, S. (2015). Performance and Quality Assessment of Similarity Measures in Collaborative Filtering Using Mahout. Procedia Computer Science, 50:229-234.
Bobadilla, J., Ortega, F., Hernando, a., and Gutiérrez, a. (2013). Recommender systems survey. KnowledgeBased Systems, 46:109-132.

Domingues, M. A., Leal, J. P., Jorge, A. M., Soares, C., and Machado, P. (2008). A platform to support web site adaptation \& monitoring of its effects: a case study. Proceedings of the 6th Workshop on intelligent techniques for web personalization \& recommender systems (ITWP 08), (Mdc):29-36.

Ekstrand, M., Ludwig, M., Konstan, J., and Riedl, J. (2011). Rethinking the Recommender Research Ecosystem: Reproducibility, Openness, and LensKit. In $A C M$ Conference on Recommender Systems, pages 133140.

Félix, C., Soares, C., Jorge, A., and Vinagre, J. (2014). Monitoring Recommender Systems: A Business Intelligence Approach. Computational Science and Its Applications-ICCSA 2014, pages 277-288.

Gantner, Z., Rendle, S., Freudenthaler, C., and SchmidtThieme, L. (2011). MyMediaLite: A Free Recommender System Library. In ACM Conference on Recommender Systems, pages 305-308.

Hahsler, M. (2011). recommenderlab: A Framework for Developing and Testing Recommendation Algorithms. Nov, pages 1-37.

Herlocker, J. L., Konstan, J. a., Terveen, L. G., and Riedl, J. T. (2004). Evaluating collaborative filtering recommender systems. ACM Transactions on Information Systems, 22(1):5-53.

Hu, Y., Koren, Y., and Volinsky, C. (2008). Collaborative Filtering for Implicit Feedback Datasets. In IEEE International Conference on Data Mining, pages 263 272.

Koren, Y. (2010). Factor in the Neighbors: Scalable and Accurate Collaborative Filtering. ACM Transactions on Knowledge Discovery from Data, 4(1):1-24.

Owen, S., Anil, R., Dunning, T., and Friedman, E. (2011). Mahout in Action. Manning Publications Co., Greenwich, CT, USA.

Said, A. and Bellogín, A. (2014). RiVal - A Toolkit to Foster Reproducibility in Recommender System Evaluation. ACM Conference on Recommender Systems.

Sarwar, B., Karypis, G., Konstan, J., and Riedl, J. (2001). Item-Based Collaborative Filtering Recommendation Algorithms. In International Conference on World Wide Web, pages 285-295.

$\mathrm{Su}$, X. and Khoshgoftaar, T. M. (2009). A Survey of Collaborative Filtering Techniques. Advances in Artificial Intelligence, 2009:1-19.

Tintarev, N. and Masthoff, J. (2011). Recommender Systems Handbook, volume 54

Vanschoren, J., van Rijn, J. N., Bischl, B., and Torgo, L. (2014). OpenML: networked science in machine learning. ACM SIGKDD Explorations Newsletter, 15(2):49-60.

Wei, K., Huang, J., and Fu, S. (2007). A survey of ecommerce recommender systems. In International Conference on Service Systems and Service Management, pages 734-738. 